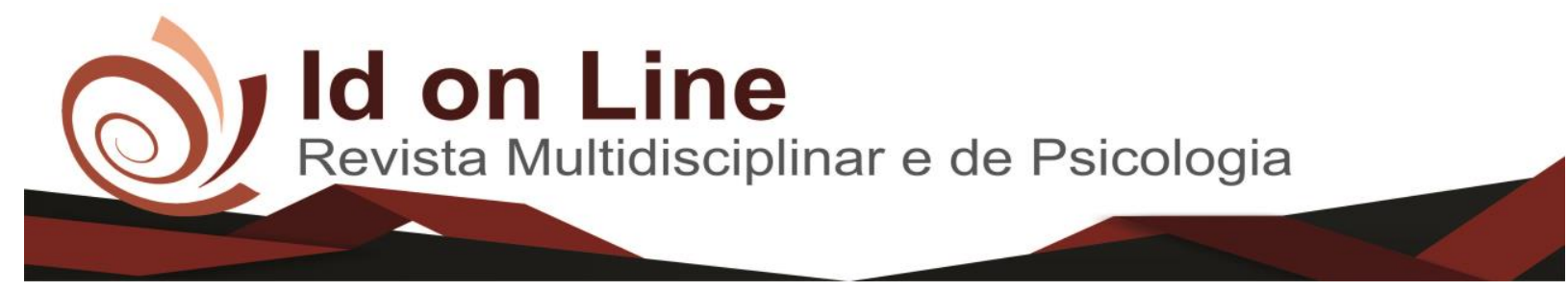

Artigo

\title{
Fatores Relacionados ao Afastamento de Enfermeiros em um Hospital Público do Município de Vitória da Conquista
}

\author{
Luana Maria de Sousa Varges ${ }^{1}$; Mirella Cristina Letto Barbosa ${ }^{2}$
}

\begin{abstract}
Resumo: A Saúde e a Segurança do Trabalho tem sido alvo de muitas discussões nos últimos anos. Atualmente tem-se observado aumento crescente no absenteísmo principalmente de profissionais que trabalham em âmbito hospitalar. Assim, este trabalho teve por objetivo analisar as principais causas de absenteísmo entre enfermeiros de um hospital público do sudoeste da Bahia no ano de 2016. Trata-se de um estudo de natureza quantitativa, transversal e descritiva que foi realizado em um hospital da rede pública de um município do sudoeste da Bahia de nível secundário, considerado de grande porte. Os dados foram obtidos a partir do banco de dados do RH e do Serviço de Saúde do Trabalhador desse hospital, organizado no programa Excel/2010. Os resultados mostraram altas taxas de absenteísmo no sexo feminino (82,9\%), a local de trabalho com maior percentual de atestados foi a UTI $(31,3 \%)$, a faixa etária com maior percentual de atestados foi a de 28 - 39 anos $(59,6 \%)$, com relação ao período de afastamento do serviço verificou-se a maior incidência de 1 à 9 dias (74\%), a causa segundo CID 10, prevaleceu doenças respiratórias $(15,8 \%)$. Conclui-se que o absenteísmo na classe de enfermagem é grande e merece atenção. Ocorre principalmente entre as mulheres, e em setores hospitalares "fechados", como a urgência/emergência e a Unidade de terapia Intensiva (UTI), na causa dos afastamentos do trabalho, é predominante às doenças do aparelho respiratório, assim como doenças osteomusculares que são impactantes na saúde dos trabalhadores de enfermagem. Considera-se relevante que outros estudos com esta temática sejam desenvolvidos, uma vez que o absenteísmo nas instituições hospitalares é grande e repercute de forma direta na qualidade da assistência que é prestada pela equipe de enfermagem ao paciente.
\end{abstract}

Palavras-Chave: Absenteísmo; Enfermagem; Trabalho.

\section{Factors Relating to the Removal of Nurses at a Public Hospital in the Municipality of Vitória da Conquista}

\begin{abstract}
Health and safety at work has been the subject of much discussion in recent years. Currently, there has been an increase in absenteeism, especially among professionals working in hospitals. Thus, this study aimed to analyze the main causes of absenteeism among nurses at a public hospital in southwest Bahia in the year 2016. It is a quantitative, cross-sectional and descriptive study that was performed in a public hospital of a municipality of southwest of Bahia of secondary level, largely considered. Data were obtained from the HR database and the Worker's Health Service of this hospital, organized in the Excel / 2010 program. The results showed high rates of absenteeism in the female sex (82.9\%), the work place with the highest percentage of certificates was the ICU (31.3\%), the age group with the highest percentage of attestations was $28-39(59.6 \%)$, the highest incidence from 1 to 9 days (74\%), the cause according to ICD 10, respiratory diseases (15.8\%) prevailed. It is concluded that absenteeism in the nursing class is great and deserves attention. It occurs mainly among women, and in "closed" hospital sectors, such as urgency / emergency and Intensive Care Unit (ICU), in the cause of withdrawal from work, is predominant to diseases of the respiratory system, as well as musculoskeletal diseases that are impact on the health of nursing workers. It is considered relevant that other studies with this theme are developed, since absenteeism in hospital institutions is large and has a direct impact on the quality of care provided by the nursing team to the patient.
\end{abstract}

Keywords: Absenteeism; Nursing; Work.

\footnotetext{
${ }^{1}$ Graduação de Enfermagem pela Faculdade Independente do Nordeste;

${ }^{2}$ Faculdade Independente do Nordeste.
} 


\section{Introdução}

A saúde e segurança do trabalhador tem sido alvo de muitas discussões nos últimos anos. A Organização Pan-Americana de Saúde (OPAS) apontou como ações primordiais e prioritárias para a saúde do trabalhador: a educação acadêmica, a formação profissional, a remuneração adequada, boas condições de trabalho, políticas de apoio, afirmando que sem isso seria impossível alcançar objetivos de desenvolvimento do Milênio (OPAS, 2015).

O cenário das instituições de saúde em que os trabalhadores estão inseridos atualmente,é complexo, com condições insalubres e inadequadas. Apesar de haver esforços para as melhorias das condições de trabalho e remuneração, percebe-se que existem diversas fragilidades no sistema, de modo amplo que impedem o pleno desenvolvimento de ações por parte dos gestores em relação aos profissionais que estão na ponta desenvolvendo o trabalho (DALRI ET al, 2014).

Atualmente as instituições de saúde tem característica de serviço intensificado, como explica Dalriet al (2014), o arranjos dos serviços ditam o aumento da produtividade e da rotatividade, através da carga horária alta devido baixo quantitativo de pessoal, a redução das horas de descanso durante a jornada laboral, colaboram para uma tendência do desenvolvimento de riscos ocupacionais, que se persistirem por um período, posteriormente podem ocasionar as doenças ocupacionais nestes trabalhadores (DALRI, et al, 2014)

Nesta perspectiva de trabalho "intensificado" e insalubre, muitos estudos têm sido desenvolvidos acerca do estresse profissional, e suas repercussões na qualidade de vida e no absenteísmo dos trabalhadores. O absenteísmo na classe da enfermagem é grave e preocupante, pois desorganiza a lógica do serviço, causa insatisfação e sobrecarga nos trabalhadores que estão presentes na instituição e consequentemente a qualidade do serviço prestado ao cliente é afetada (SELEGHIM, 2012; GOMES et al., 2007).

O absenteísmo é definido como a ausência/falta no ambiente de trabalho, devido diversas causas como patologias, acidentes ocupacionais e direitos legais como (ALMEIDA et al, 2015).

Para Costa et al (2014), o absenteísmo vem se tornando um problema que merece atenção por meio das instituições de saúde.Atualmente tem-se observado aumento crescente no absenteísmo principalmente de profissionais que trabalham em âmbito hospitalar. "O absenteísmo é um importante indicador de avaliação da saúde dos trabalhadores e das condições em que o trabalho é realizado, como também da política de recursos humanos da instituição e do serviço de atenção à saúde do trabalhador" (UMANN, et al, 2011 p 184). 
O interesse em realizar este estudo se deu após cursar a disciplina "saúde do Trabalhador" e presenciar absenteísmo durante os estágios curriculares. Observa-se a importância de estudos que demonstrem o quantitativo de trabalhadores de enfermagem que, por motivos de saúde, precisam se ausentar do trabalho, tendo em vista o problema gerado em torno deste afastamento tanto para os outros trabalhadores, paciente e instituição de saúde como a diminuição da qualidade dos cuidados prestados aos clientes, para enfim poder buscar soluções cabíveis. Portanto, o objetivo deste estudo foi analisar as principais causas de absenteísmo entre enfermeiros de um hospital público do sudoeste da Bahia no ano de 2016.

\section{Metodologia}

Trata-se de uma pesquisa de natureza quantitativa, transversal e descritiva. A pesquisa foi realizada em um hospital da rede pública de um município do sudoeste da Bahia de nível secundário, considerado de grande porte. As pesquisas quantitativas têm os resultados matematicamente "contados" quantificados, assim, pesquisas deste tipo são objetivas uma vez que descrevem os fenômenos e a realidade a partir da quantificação dos mesmos (GIL, 2010).

Os dados foram obtidos a partir do banco de dados do setor de Recursos Humanos (RH)e do Serviço de Saúde do Trabalhador (SST) desse hospital. Os dados são organizados no programa Excel/2010, e contém informações referentes ao sexo, idade, estado civil, número de vínculos empregatícios, setor de trabalho, data de admissão no hospital, motivo de afastamento e tempo do afastamento.

A coleta de dados ocorreu por meio de análise retrospectiva de ficha do funcionário no RH e de atestados médicos apresentados no ano de 2016. A coleta de dados foi realizada na primeira quinzena de setembro de 2017.

Os dados após analisados e organizados, foram apresentados em forma de tabela de forma a facilitar a análise descritiva dos mesmos utilizando-se números absolutos e porcentagens. A definição das causas de afastamento levou em consideração a Classificação Internacional de Doenças e Problemas Relacionados à Saúde (CID 10).

Para a realização da pesquisa foram observadas todas as normas da Resolução n 466/12 do Conselho Nacional de Saúde para pesquisas envolvendo seres humanos. Portanto, os dados foram coletados após aprovação do projeto pelo Comitê de Ética e Pesquisa (CEP) da Faculdade Independente do Nordeste. Obtendo o parecer favorável sob o número: 72175317.7.0000.5578. 


\section{Resultados}

Tabela 1 - Percentual de atestados emitidos por enfermeiros em uma Unidade Hospitalar segundo o sexo, 2016.

\begin{tabular}{ccc}
\hline GÊNERO & $\mathbf{N}^{\circ}$ & $\%$ \\
\hline FEMININO & 121 & $82.9 \%$ \\
MASCULINO & 25 & $17.1 \%$ \\
TOTAL & 146 & $100 \%$ \\
\hline
\end{tabular}

FONTE: Coleta de dados no SIAST (HGVC) 2016

Na tabela 1 observa-se que o quantitativo de atestados emitidos no ano de 2016 foram de 146 atestados, sendo que $121(82,9 \%)$ eram de profissionais do sexo feminino e $25(17,1 \%)$ do sexo masculino.

Tabela 2: Percentual de atestados emitidos por enfermeiros em uma Unidade Hospitalar segundo o local de trabalho, 2016.

\begin{tabular}{|c|c|c|}
\hline SETORES & № DE ATESTADOS & $\%$ \\
\hline EMERGENCIA & 30 & $20,5 \%$ \\
\hline CLINICA CIRÚRGICA & 23 & $15,8 \%$ \\
\hline UTI 1 & 31 & 21,3 \\
\hline CLINICA MÉDICA & 21 & $14,3 \%$ \\
\hline PEDIATRIA & 9 & $6,2 \%$ \\
\hline UTI NEONATAL & 6 & $4,1 \%$ \\
\hline SALA DE CHOQUE & 6 & $4,1 \%$ \\
\hline OUTROS & 20 & 13,7 \\
\hline TOTAL & 146 & $100 \%$ \\
\hline
\end{tabular}

FONTE: Coleta de dados no SIAST (HGVC) 2016

A tabela 2 apresenta o quantitativo de atestados dados pelos enfermeiros distribuídos por setores da unidade hospitalar apresentando $20,5 \%$ nos setores de emergência e 31,3\% na 
UTI, segredos da clinicas cirúrgica com $15,8 \%$ e clínica médica com 14,3\% da emissão dos atestados.

Tabela 1: Percentual de atestados emitido emitidos por enfermeiros em uma Unidade Hospitalar de acordo a idade dos profissionais enfermeiros, 2016.

\begin{tabular}{|c|c|c|}
\hline IDADE EM ANOS & № DE ATESTADOS & $\%$ \\
\hline $28-39$ & 87 & 59,6 \\
\hline $40-49$ & 30 & 20,5 \\
\hline $50-59$ & 19 & 13 \\
\hline IGNORADOS & 10 & 6,9 \\
\hline TOTAL & 146 & 100 \\
\hline
\end{tabular}

FONTE: Coleta de dados no SIAST (HGVC) 2016

A tabela 3 demonstra o percentual de atestados emitidos de acordo com a idade do profissional, onde observa-se que 59,6\% são de profissionais com idade entre 28-39 anos e um percentual pequeno de $13 \%$ em profissionais com 50 a 59 anos de idade.

Tabela 4: Distribuição percentual dos atestados emitidos por enfermeiros em uma Unidade Hospitalar conforme os dias de afastamento, 2016.

\begin{tabular}{|c|c|c|}
\hline DIAS AFASTADOS & № DE ATESTADOS & $\%$ \\
\hline $1-9$ & 108 & $74 \%$ \\
\hline $10-19$ & 26 & $17,8 \%$ \\
\hline $20-29$ & 2 & $1,4 \%$ \\
\hline $30-90$ & 5 & $3,4 \%$ \\
\hline TEMPO INDETERMINADO & 1 & $0,7 \%$ \\
\hline IGNORADOS & 4 & $2,7 \%$ \\
\hline TOTAL & 146 & $100 \%$ \\
\hline
\end{tabular}

FONTE: Coleta de dados no SIAST (HGVC) 2016 
A tabela 4 apresenta o tempo de afastamento dos profissionais no trabalho onde $74 \%$ dos atestados são de 1 a 9 dias de afastamento seguido de 17,8\% de 10 a 19 dias e de 3,4\% para períodos maiores entre 30 a 90 dias.

Tabela 5: Distribuição percentual de atestados emitidos por enfermeiros em uma UH segundo o grupo de causas de doenças (CID10), 2016.

\begin{tabular}{lcc}
\multicolumn{1}{c}{ GRUPO DE CAUSAS DE DOENÇAS } & No DE & $\%$ \\
& ATESTADOS & \\
\hline DOENÇAS DO APARELHO RESPIRATORIO & 23 & $15,8 \%$ \\
\hline DOENÇAS OSTEOMUSCULARES & 16 & $11 \%$ \\
\hline DOENÇAS DO APARELHO DIGESTIVO & 14 & $9,6 \%$ \\
\hline DOENÇAS INFECCIOSAS E PARASITARIAS & 10 & $6,8 \%$ \\
\hline ABDOME AGUDO & 11 & $7,5 \%$ \\
\hline CAUSAS EXTETERNAS & 11 & $7,5 \%$ \\
\hline ANORMALIDADES NO PERIODO GESTACIONAL & 9 & $6,2 \%$ \\
DOENÇAS MENTAIS E COMPORTAMENTAIS & 7 & $4,8 \%$ \\
\hline DOENÇAS DAS MUCOSAS OCULARES & 6 & $4,1 \%$ \\
\hline DOENÇAS DO APARELHO CIRCULATORIO & 6 & $4,1 \%$ \\
\hline DOENÇAS DO APARELHO RESPIRATORIO & 5 & $3,4 \%$ \\
\hline NEOPLASIAS & 1 & $0,7 \%$ \\
\hline OUTRAS CAUSAS & 12 & $8,2 \%$ \\
\hline IGNORADOS & 15 & $10,3 \%$ \\
\hline TOTAL & 146 & $100 \%$ \\
\hline
\end{tabular}

FONTE: Coleta de dados no SIAST (HGVC) 2016

A tabela 5 apresenta as causas do absenteísmo, observados nos atestados apresentados pelos enfermeiros no período de janeiro a dezembro de 2016, sendo agrupados de acordo com a classificação internacional de doenças - CID10. As principais causas observadas no afastamento dos profissionais enfermeiros correspondem a 15,8\% das DAR e Doenças Osteomusculares com $11 \%$ das causas. Observa-se também que 10,3\% dos atestados foram de causas sem identificação. 
Tabela 6: Distribuição percentual de atestados emitidos por enfermeiros em uma UH de acordo o setor e sexo em 2016.

\begin{tabular}{|c|c|c|c|c|}
\hline \multirow{3}{*}{ SETOR } & \multicolumn{4}{|c|}{ SEXO } \\
\hline & \multicolumn{2}{|c|}{ FEMININO } & \multicolumn{2}{|c|}{ MASCULINO } \\
\hline & № & $\%$ & $\mathrm{~N}$ & $\%$ \\
\hline EMERGENCIA & 30 & 20,5 & 0 & 0 \\
\hline UTI 1 & 25 & 17,1 & 6 & 4,1 \\
\hline CLINICA CIRURGICA & 20 & 13,7 & 3 & 2 \\
\hline CLINICA MÉDICA & 12 & 8,2 & 9 & 6,2 \\
\hline PEDIATRIA & 7 & 4,7 & 2 & 1,4 \\
\hline UTI NEONATAL & 5 & 3,4 & 1 & 0,7 \\
\hline SALA DE CHOQUE & 5 & 3,4 & 1 & 0,7 \\
\hline ACOLHIMENTO & 4 & 2,7 & 0 & 0 \\
\hline OUTROS & 13 & 8,9 & 3 & 2 \\
\hline TOTAL & 121 & 82,9 & 25 & 17,1 \\
\hline
\end{tabular}

FONTE: Coleta de dados no SIAST (HGVC) 2016

Na tabela 6 aponta a quantidade de atestados apresentados por enfermeiros distribuídos de acordo o setor e sexo. Observa-se que 20,5\% dos atestados foram emitidos pelo sexo feminino e no setor da emergência, seguidos de 17,1\% na UTI e 13,7 na clínica cirúrgica, todos os atestados emitidos por enfermeiras. Observa-se ainda que 6,2 \% são atestados emitidos por enfermeiros do sexo masculino e do setor da clinica médica.

\section{Discussões}

Para a discussão dos dados encontrados, foram criados dois eixos temáticos, relacionados entre si: O primeiro eixo é intitulado-“Absenteísmo avaliado a partir de gênero e local de trabalho" e segundo eixo-“tempo do afastamento do trabalho e causas". 


\section{Absenteísmo avaliado a partir de gênero, idade e local de trabalho}

O absenteísmo foi observado com grande incidência nos profissionais de sexo feminino $(82,9 \%)$, como mostra a tabela 1 . Uma pesquisa realizada pelo Conselho Federal de Enfermagem (COFEN), em 2011, mostrou resultados que apontaram para a região Nordeste do Brasil com o maior quantitativo de profissionais mulheres, com porcentagem de 90,08\% dos profissionais. O quantitativo de enfermeiros do sexo feminino é extremamente maior quando comparado ao masculino (COFEN, 2011).

Martins e Scherer (2016) realizou um estudo descritivo, exploratória, quali-quantitativo em um Hospital Universitário de Brasília, no setor de emergência de um hospital com o intuito de avaliar o absenteísmo em diversos aspectos e que tange ao gênero, os dados mostraram que $88 \%$ dos atestados eram oriundos de mulheres, condizente com os dados deste presente estudo.

O estudo de Ferreira et al (2012), traz resultados semelhantes aos aqui encontrados com taxas de absenteísmo mais altas nos profissionais do sexo feminino, quando comparados aos do sexo masculino. Para Rissardoet al (2013), a alta prevalência de mulheres está relacionada a cultura de que cuidado é realizado por mulheres, construção histórica da feminilização do cuidado que ainda prevalece até os dias atuais.

Costa et al (2014) corrobora com este pensamento e diz que na construção da história da Enfermagem, todos os cuidados que eram prestados aos doentes, eram feitos por mulheres em quase todas as situações. Nos dias atuais, a predominância do gênero feminino ainda é grande e o absenteísmo nesta classe também é atrelado devido a sobrecarga da mulher no local de trabalho e também com os trabalhos domésticos.

Para além da parte histórica que é atrelada ao gênero feminino, atualmente as mulheres conquistaram muitos espaços na sociedade, hoje, no Brasil, cerca de $38 \%$ das mulheres são responsáveis pelo provimento e sustento das famílias. As mulheres vivem mais que a população masculina, no entanto adoecem mais que eles (IBGE, 2011).

A inserção da mulher no mundo de trabalho, a partir do atual contexto da globalização economia, as reestruturações produtivas, verificadas a partir da lógica de flexibilização, criam novos rumos para a organização do trabalho e repercutem em mudanças no mercado de trabalho, as desigualdades relacionadas ao gênero são observadas principalmente a partir de variações no trabalho pelo sexo e mão de obra (BRASIL, 2017).

A mulher se posiciona no mundo de trabalho, secundarizada com base na sua posição assumida na família. É sabido que as mulheres utilizam o dobro do tempo nas atividades 
domésticas quando comparada aos homens, contabilizando ao longo da semana, as mulheres trabalham cerca de cinco horas mais que eles. Além da jornada dupla de trabalho, alternada entre lar e local de trabalho, as mulheres adoecem mais em função de assédios (moral e sexual), sofridos no âmbito de trabalho, tanto em repartições públicas, quanto privadas (BRASIL, 2017).

Com relação a faixa etária, a idade predominante neste estudo foi 28 à 39 anos (59,6\%), como mostra a tabela 3. O estudo de Lopes (2015), traz dados compatíveis ao deste estudo, com relação a idade dos profissionais que apresentavam maiores taxas de absenteísmo, no estudo de Lopes (2015) ,a faixa etária foi a de 31 a 35 anos obteve maiores índices de absenteísmo.

Ressalta-se que apesar das outras faixas etárias apresentarem baixas incidências, de acordo Ferreira et al (2012), idades mais avançadas associou-se, bem como servidores públicos, indivíduos casados ou separados, divorciados ou viúvos apresentam chances elevadas de terem muitos dias de ausência.

Com relação ao setor de emergência, Ferreira et al (2012), traz que este e um ambiente agitado, em que os profissionais tem muitos pacientes sob sua responsabilidade, sob seus cuidados, além da média de diária alta de pacientes, o setor de urgência e emergência tem alta rotatividade de pacientes e não existe numero determinado de leitos, principalmente em instituições públicas, o autor ainda complementa que pela baixa remuneração dos profissionais, os mesmos são obrigados a possuir mais de um emprego, o que consequentemente leva à sobrecarga física, emocional, psíquica e por fim o absenteísmo.

Lopes (2015) traz em sua pesquisa relatos de profissionais dos setores de urgência e emergência, que justificaram as ausências devido o estresse a que os profissionais estão submetidos e o despreparo em lidar com essa situação. Além dos fatores estressores do próprio ambiente, apontados no parágrafo anterior, outros estressores contribuem para o absenteísmo e são eles: modalidade de trabalho noturno, atritos no trabalho, a forma de organização dos profissionais nas unidades hospitalares, confronto constante com a morte, injúrias, riscos, e ainda a falta de autonomia do profissional mediante determinadas situações (LOPES, 2015; FERREIRA et al, 2016).

Para além do absenteísmo no trabalho, o estresse decorrente do trabalho ocasionado no setor de emergência aumenta a incidência da ocorrência da Síndrome de Burnoutentre os profissionais da enfermagem. Para Nascimento et al (2013) esta síndrome caracteriza-se por meio da presença de sintomas físicos, psíquicos, comportamentais e defensivos. 
A Síndrome de Burnouté tida como a síndrome do profissional descuidado, pois nelao trabalhado fica "perdido" e não consegue enxergar mais sentido nas relações produtivas de trabalho, como verifica-se na fala de Rissardo e Gasparino (2013):

\footnotetext{
profissional perde o sentido da sua relação com o trabalho e sente como se as coisas já não tivessem mais importância" Trata-se de um conceito que envolve três dimensões, que podem aparecer associadas, mas que são independentes: exaustão emocional; despersonalização; e falta de envolvimento no trabalho ou diminuição da realização pessoal (RISSARDO e GASPARINO 2013, p.129)
}

Em busca de avaliar a presença de sintomas da síndrome, um estudo realizado por Pereira et al (2014) em um hospital de urgência/emergência avaliou 40 profissionais de enfermagem através da utilização de um roteiro intitulado MaslachBurnoutInventory, com intuito de diagnosticar sintomas da Síndrome de Burnout. Os resultados mostraram que mais de $80 \%$ dos profissionais apresentavam sentimento de incompetência no trabalho, de forma moderada. As discussões dos autores apontam que mesmo a presença moderada de sintomas já caracteriza inicio da síndrome, uma vez que o desequilíbrio entre os profissionais e o local de trabalho confirma o desgaste profissional.

\section{Tempo de afastamento do trabalho e causas}

O estudo de Martins e Scherer (2016), mostrou que a média de afastamento do trabalho foi de 6,27 dias. Os resultados do atual estudo também são semelhantes, mostrando que a duração do afastamento do trabalho fica em torno de 1 à 9 dias (74\%), como mostrado na tabela numero 4. Os autores apontam que os atestados com período de afastamento, mairo que 30 dias tem aumentado significativamente ao longo dos últimos anos (MARTINS e SCHERER, 2016).

Dentre os motivos relacionados ao absenteísmo, Lopes (2015) mostrou em seu estudo que a sobrecarga de trabalho ao desgaste excessivo, estresse e esgotamento físico como um dos principais fatores que levavam ao absenteísmo na instituição.

A presente pesquisa traz as causas de absenteísmo, de acordo o laudo do atestado que era baseado na Classificação Internacional das Doenças (CID10), assim a causa base que teve maior incidência e levava ao absenteísmo foram as doenças do aparelho respiratório com percentual de quase 16\%, como visualiza-se na tabela 5. O estudo de Costa et al (2014) traz dados semelhantes e mostra que as principais causas de absenteísmo na equipe de enfermagem 
são licenças médicas devido doenças do sistema osteomuscular, doenças do aparelho respiratório, doenças comportamentais e doenças mentais.

O autor supracitado também traz uma crítica ao processo de trabalho em que os profissionais de saúde estão inseridos, onde a predominância de ambientes insalubres para os profissionais desenvolverem suas atividades é grande, além de diversas condições de trabalho inadequadas e exposição a vários tipos de riscos. Essa teia de problemas afeta de forma direta a saúde dos trabalhadores, levanto ao desencadeamento de desgastes, injurias e patologia que causam o absenteísmo (COSTA et al, 2014).

Um estudo quantitativo descritivo, realizado por Pires et al (2003), objetivou identificar as causas de absenteísmo por doença entre os trabalhadores de Enfermagem do de um Hospital Universitário de São Paulo, mostrou que no período de 11 meses um total de 67 licenças médicas levaram a 1033 dias perdidos de trabalho. As causas dos afastamentos do serviço foram em primeiro lugar doenças osteomusculares e em segundo lugar, doenças do tecido conjuntivo.

As doenças osteomusculares ou Distúrbios Osteomusculares Relacionados ao Trabalho (DORT) compreendem um grande e heterogêneo grupo de agravos crônicos do sistema musculoesquelético, devido desenvolvimento de atividades realizadas durante o trabalho. As atividades laborais acometem de forma negativa os componentes do sistema osteomuscular, o quadro clínico das DORTs é variável com queixas de parestesia, dor e fadiga. As DORTs consistem em um dos problemas mais recorrentes e dispendiosos na classe de enfermagem gerando a incapacidade e absenteísmo no serviço (OLIVEIRA e ALMEIDA, 2017).

O Absenteísmo no local de trabalho constitui-se um problema não só no Brasil, mas também em países comoa Pensilvânia, Castle e Ferguson-Rome (2015), trazem em seu trabalho que muitos profissionais de enfermagem trabalham em dois ou mais serviços de saúde, além de terem ocupações e desafios pessoais. Os autores pontuam que a os profissionais predominantes são mulheres e dentre estes desafios pessoais estão: problemas com transporte, maternidade solteira e ter que cuidar de outros membros da família.

Bargas e Monteiro (2014) comparando o Brasil com Canadá evidenciaram que auxiliares de Enfermagem apresentaram maior média de ausências por licença médica que os técnicos de Enfermagem e estes, mais que os enfermeiros, no entanto o estudo não conseguiu evidenciar se as doenças eram ocupacionais ou motivacionais. Em comparação no Canada enfermeiros que tinham salários menores e faixa etária mais avançada, eram mais propensos a apresentarem absenteísmos. 
De acordo Silveira (2009) os altos índices de absenteísmos dos profissionais de enfermagem não podem ser explicados apenas levando em consideração apenas os baixos salários, mas também as condições de trabalho de forma global. As causas do absenteísmo são diversas, e o autor pontua que tanto no Brasil, como em outros países como a Inglaterra, as altas taxas de absenteísmo são ocasionadas por problemas osteomusculares ou problemas mentais e psicológicos, discussões condizentes com os dados encontrados no atual estudo.

Nesta lógica, como o absenteísmo é relacionado diretamente com o aumento ou a diminuição dos custos financeiros da empresa, esse indicador mostra de que forma a instituição está em nível de competitividade no mercado. Haja vista que ausência dos profissionais interfere no modo como os serviços são prestados e também no resultado final de todo esse processo. Assim, estrategicamente falando é interessante que as instituições de saúde proporcionem melhores condições de trabalho para os profissionais (RIBEIRO e SOUZA, 2016).

\section{Considerações Finais}

A partir das discussões levantadas acima e dos dados coletados, pode-se concluir que o estudo respondeu ao objetivo proposto incialmente, mostrando o absenteísmo na classe de enfermagem é grande e merece atenção. Especialmente entre as mulheres, haja vista o grande quantitativo de profissionais pertencentes a este gênero.

O absenteísmo como observado nos dados e em outras pesquisas é mais incidente em setores hospitalares "fechados", como a urgência/emergência e a Unidade de terapia Intensiva (UTI), ambientes em que o grau de estresse dos profissionais é maior quando comparado a outros setores. No que tange à causa dos afastamentos do trabalho, observou-se prevalência de doenças do aparelho respiratório, assim como doenças osteomusculares que são impactantes na saúde dos trabalhadores de enfermagem.

Por fim, considera-se relevante que outros estudos com esta temática sejam desenvolvidos, uma vez que o absenteísmo nas instituições hospitalares é grande e repercute de forma direta na qualidade da assistência que é prestada pela equipe de enfermagem ao paciente. 


\section{Referências}

ALMEIDA, DRO de; NASCIMENTO, IGN do; NETO, JMS; ALMEIDA, AG de B. CAUSAS E DESVANTAGENS DO ABSENTEÍSMO: O Caso da Empresa Auto Center 24 Horas em Porto Velho. Congresso Nacional de Excelência em gestão. 13 e 14 de agosto de 2015. ISSN 1984-9354.

BARGAS, EB; MONTEIRO, MI. Fatores relacionados ao absenteísmo por doença entre trabalhadores de Enfermagem. Acta Paul Enferm. 2014; 27(6):533-8.

BRASIL. Instituto Brasileiro de Geografia e Estatística - IBGE. Algumas das principais características dos Trabalhadores Domésticos vis a vis a População Ocupada. Pesquisa Mensal de Emprego (PME): 2011.

BRASIL, Documento Orientador da $2^{\mathrm{a}}$ Conferência Nacional de Saúde das Mulheres: Saúde das mulheres - desafios para a Integralidade com equidade. Conselho Nacional de Saúde. Brasília, 2017.

CASTLE, N; FERGUSON-ROME, JC.Influence of Nurse Aide Absenteeism on Nursing Home Quality.The Gerontologist, 2015, Vol. 55, No. 4

COFEN. Comissão de Business Intelligence - Produto 2: Análise de dados dos profissionais de enfermagem existentes nos Conselhos Regionais, 2011. Disponível em: http://www.cofen.gov.br/wp-content/uploads/2012/03/pesquisaprofissionais.pdf acesso em 20/10/2017

CONFEDERAÇÃO NACIONAL DOS TRABALHADORES EM SAÚDE. Jornal da CNTS. Brasília/ DF. Ano 18 - N 76, 2013.

DALRI, RC de MB; da SILVA, LA;MENDES, AMOC; ROBAZZI, MRCC . Carga horária de trabalho dos enfermeiros e sua relação com as reações fisiológicas do estresse. Rev. LatinoAm. Enfermagem nov.-dez. 2014;22(6):959-65.

FERREIRA, R. C.; GRIEP, R. H.; FONSECA, M. J. M.; ROTENBERG, L. Abordagem multifatorial do absenteísmo por doença em trabalhadores de enfermagem. Rev Saúde Pública. v.46, n.2, p:259-68, 2012.

FORTE, ECN; TROMBETTA, AP; de PIRES, DEP; GELBCKE, FL de; LINO, MM. Abordagens teóricas sobre a saúde do trabalhador de enfermagem: revisão integrativa. CogitareEnferm. 2014; 19(3):604-11

GOMES, A. R. et al. Absenteísmo de enfermagem em hospital público universitário. Revista do Instituto de Ciências da Saúde, São Paulo, v. 25, n. 3, p. 213-219, 2007.

GONÇALVES, FGA; SOUZA, NVDO; PIRES, AS da; SANTOS, DM dos; D’OLIVEIRA, CAFB; RIBEIRO, LV. Modelo neoliberal e suas implicações para a saúde do trabalhador de enfermagem. Revenferm UERJ, Rio de Janeiro, 2014 jul/ago; 22(4):519-2 
Lei No. 8080/90, de 19 de setembro de 1990. Brasília: DF. 1990. Disponível em https://www.planalto.gov.br/ccivil_03/LEIS/L8080.htm

LOPES, M. C. Absenteísmo-doença na enfermagem: caracterização das ocorrências em um hospital de ensino. Trabalho de Conclusão de Curso, da Universidade de Santa Cruz do Sul UNISC, para a obtenção do título de Bacharel em Enfermagem, 2015

MARTINS, N. R. A. V.; SCHERER, M. D. A. Análise do absenteísmo por doença em uma unidade de urgência e emergência do Hospital Universitário de Brasília. Disponível em http://bdm.unb.br/bitstream/10483/15495/1/2016_NataliaReginaAlvesVazMartins_tcc.pdf

NASCIMENTO, C. C.; CHAVES, G. B. O.; MELO, J. E. A.; SOUZA, W. L.; FIREMAN, E. F. Conhecimento publicado acerca do absenteísmo relacionado à síndrome de bournout com os profissionais de enfermagem: revisão integrativa. Cadernos de Graduação - Ciências Biológicas e da Saúde. v.1, n.2, p. 121-134, 2013.

OPAS. Cúpula sobre Desenvolvimento Sustentável, 25-27 de setembro, sede das NU, Nova York, NY, EUA. United NationsSustainableDevelopmentSummit 2015.

OLIVEIRA VC, ALMEIDA RJ. Aspectos que Determinam as Doenças Osteomusculares em Profissionais de Enfermagem e seus Impactos Psicossociais. J Health Sci 2017;19(2):130-

PIRES, A.V.C.; MAGALHÃES, C. C.; MENEZES, L. C. M.; COSTA, M. F. L. Absenteísmo por doença na equipe de enfermagem do Hospital Universitário Unidade Presidente Dutra. Disponível em: http://apps.cofen.gov.br/cbcenf/sistemainscricoes/arquivosTrabalhos/absenteismo\%20por\%20 doenca\%20n.pdf acesso em: 03/11/2017

REGIS, F.; VEIGA JUNIOR, CL da. Acidente de trabalho: Responsabilidade civil do empregador. Revista Eletrônica de Iniciação Científica. Itajaí, Centro de Ciências Sociais e Jurídicas da UNIVALI. 4(1):768-784, 2013.

RIBEIRO, R. N.; SOUZA, P. M. Relação entre qualidade de vida no trabalho e o índice de absenteísmo nas organizações: uma análise empírica do absenteísmo em profissionais de enfermagem. II CONGRESSO DE ADMINISTRAÇÃO E TECNOLOGIA - CAT O Estudo das Tecnologias frente às mudanças sociais 7 a 11 de novembro de 2016.

RISSARDO, M.P.; GASPARINO, R.C. Exaustão emocional em enfermeiros. Esc Anna Nery (impr.); v.17, n.1, p:128 - 132, 2013

SANTANA Vilma Sousa, SILVA da Jandira Maciel. Os 20 anos da saúde do trabalhador no Sistema Único de Saúde do Brasil: limites, avanços e desafios. Secretaria de Vigilância em Saúde/MS. 2008

SELEGHIM MR, MOMBELLI MA, OLIVEIRA MLF, WAIDMAN MAP, MARCON SS. Sintomas de estresse em trabalhadoras de enfermagem de uma unidade de pronto socorro. Rev Gaúcha Enferm. 2012;33(3):165-173.

SILVEIRA, AM. Saúde do trabalhador. Belo Horizonte: Nescon/UFMG, Coopmed, 2009. 
UMANN, J; GUIDO, L de A; LEAL, KP; FREITAS, E de O. Absenteísmo na equipe de enfermagem no contex to hospitalar. CiencCuidSaude 2011 Jan/Mar; 10(1):184-190.

\section{Como citar este artigo (Formato ABNT):}

VAEGES, Luana M. de S.; BARBOSA, Mirella C. L. Fatores Relacionados ao Afastamento de Enfermeiros em um Hospital Público do Município de Vitória da Conquista. Id on Line Revista Multidisciplinar e de Psicologia, 2017, vol.11, n.38, p.663-677. ISSN: 1981-1179.

Recebido: 07.11.2017

Aceito: 09.11.2017 